\title{
Reflexiones sobre la "trans-formación" de la universidad mediante la transdisciplinariedad en prácticas docentes e investigación
}

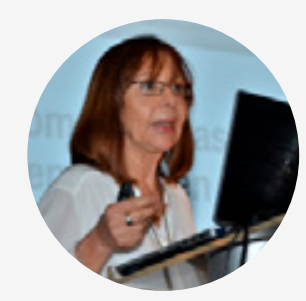

DRA. MARÍA E. CÓRDOBA*

\section{Reflections on the "trans-formation" of the university through transdisciplinarity in teaching practices and research}

Recibido: 31 de marzo de 2019 | Aprobado: 5 de junio de 2019
"Tiempos extraños son estos en que al joven y al viejo
se les enseñan falsedades en la escuela.
Y a la persona que se atreve a decir la verdad,
inmediatamente se la llama loca o tonta"

(Platón 427 AC)

\section{Resumen}

En educación superior resulta evidente la necesidad de un cambio en los planes y estrategias que los docentes implementan en sus prácticas, una renovación capaz de atender la complejidad en la que vivimos y que contemple la protección de la sociedad y el medioambiente como elementos de prioridad en el quehacer de los futuros profesionales, de manera que puedan responder congruentemente a las problemáticas actuales. La necesidad de cambio no es un descubrimiento de los últimos años, es un tema que ha estado en discusión por mucho tiempo, sin embargo, los esfuerzos e iniciativas para transitar a un nuevo modelo han resultado poco efectivos o insuficientes. El modelo educativo fundamentado en diseños estructurados exclusivamente con disciplinas desligadas unas de otras dificulta dar una respuesta integral a las complejas problemáticas sociales que se nos presentan. Por ello, las instituciones de educación superior se plantean la superación del paradigma educativo tradicional, que involucra la enseñanza separada en disciplinas (asignaturas), para dar paso a modelos transdisciplinarios, que abarcan varias disciplinas en forma transversal; no solo de nombre o con retoques a lo existente, sino hacia un enfoque que pueda trascender con un salto cualitativo. La transformación implica que desde las aulas se asuma la enseñanza abordando el sujeto u objeto de estudio de manera que se integren las diversas dimensiones y áreas del saber que lo componen, a fin de posibilitar una comprensión lo más cercana a la complejidad del mismo. Para lograrlo, debe abrirse el diálogo para la trans-formación de la educación superior, escuchando diferentes voces, no solo académicas, y que incluya todos los niveles y actores involucrados en la educación superior. La propuesta de esta reflexión sugiere la transdisciplinariedad como una forma de lograr esta transformación.

Palabras clave: transdisciplinariedad; educación superior; transformación de la universidad; integración de los conocimientos; pensamiento complejo.

\footnotetext{
* Actualmente se desempeña como Docente Investigadora en el Instituto Tecnológico de Santo Domingo y consultora independiente en materia educativa, con énfasis en los campos de Estudios Generales, transdisciplinariedad, currículo y evaluación auténtica. Cursó Psicología, Educación Social, Animación Sociocultural y Doctorado en Educación. Para contactar a la autora: macorbu@yahoo.com
}

ISSN (en línea): 1814-4152 / Sitio web: http://cuaderno.pucmm.edu.do

CÓMO CITAR: Córdoba, M. (2019). Reflexiones sobre la "trans-formación" de la universidad mediante la transdisciplinariedad en prácticas docentes e investigación. Cuaderno de Pedagogía Universitaria, Vol. 16, n. 32, julio-diciembre, pp. 100-108 


\section{Abstract}

In higher education, the need for a change in the plans and strategies that teachers implement in their practices is evident, a renewal capable of addressing the complexity in which we live and that includes the protection of society and the environment as priority elements in the work of future professionals, so that they can respond congruently to current problems. The need for change is not a discovery of recent years, it is an issue that has been under discussion for a long time, however, the efforts and initiatives to move to a new model have been either ineffective or insufficient. The educational model based on designs structured exclusively with disciplines that are separated from each other makes it difficult to give an integral response to the complex social problems that are presented to us. Therefore, institutions of higher education consider the overcoming of the traditional educational paradigm, which involves separate teaching in disciplines (subjects), to give way to transdisciplinary models, which cover several disciplines in a transversal way, not only in name or with adjustments to the existing ones, but towards an approach that can transcend with a qualitative leap. The transformation implies that, from the classrooms, the teaching is assumed by approaching the subject or object of study in a way that integrates the diverse dimensions and areas of knowledge that compose it, in order to enable an understanding as close as possible to its complexity. To achieve this, the dialogue for the trans-formation of higher education must be opened, listening to different voices, not only academics, and including all the levels and actors involved in higher education. The proposal of this reflection suggests transdisciplinarity as a way to achieve this transformation.

Keywords: transdisciplinarity; higher education; transformation of the university; knowledge integration; complex thinking.

\section{Introducción}

Desde hace décadas el futuro de la educación superior se vislumbra en la «evolución transdisciplinaria del aprendizaje». El núcleo de las reflexiones ha sido el modelo educativo transdisciplinario y las alternativas para superar los desafíos planteados por diferentes epistemologías, métodos y posiciones éticas tradicionales en torno a la educación. A pesar de estos esfuerzos, salvo casos aislados, el modelo educativo sigue influenciado mayormente por la división del saber en disciplinas independientes. Una posible razón puede estar asociada con el hecho de que, en diversos contextos universitarios, el desempeño profesional de los académicos se mide o valora más por el rendimiento de la investigación, mientras el interés y evaluación de la práctica docente no siempre reciben la misma atención y rigurosidad. Como consecuencia, el gran esfuerzo está dirigido a las publicaciones y no tanto a la incorporación de nuevos planes y estrategias docentes que contemplen la transdisiplinariedad como eje central. Aceptarlo implica reconocer que queda la gran tarea de descubrir cómo podrían las organizaciones académicas encontrar formas adecuadas e incentivos para la enseñanza y el aprendizaje transdisciplinario (Rondelli, 2015).

A lo largo de esta reflexión se presenta la problemática sobre las debilidades que enfrenta la educación superior para dar respuestas adecuadas a la complejidad e incertidumbre por la que atraviesa el contexto actual. Se presenta el modelo transdisciplinario como alternativa para superar el fragmentarismo en la educación. Además, se pretende motivar la inquietud que debe generarse en cada institución de educación superior a fin de abordar programas pilotos en los que se invierta capacitación en la docencia, investigación y evaluación en el enfoque transdisciplinar. Por último, se busca motivar el desarrollo de una educación general inter y transdisciplinaria para lograr la transformación y fortalecimiento de la universidad en el siglo XXI que supere la segmentación del saber.

\section{Problemática}

El mundo atraviesa momentos muy difíciles con problemáticas sociales y medioambientales complejas a las que el sistema educativo ha tenido dificultades para responder adecuadamente, allí nace la búsqueda de opciones para generar propuestas de cambio. Situaciones como:

el calentamiento global del planeta, la crisis del petróleo, la extinción de miles de especies, la escasez de recursos naturales indispensables para la sobrevivencia, la nueva crisis del patrón de acumulación del sistema capitalista o el cambio cultural inmenso al que ha llevado la revolución tecnológica (Pozzoli, 2006)

se encuentran dentro de las problemáticas más discutidas y relevantes; cada una de ellas compleja y abarcadora y con efectos para toda la población 
mundial. La magnitud de sus implicaciones y alcance no responden a análisis y soluciones reduccionistas, simplificadores y que fallan al no ver la totalidad del evento, ya que tratan de separarlo en partes aisladas; un tipo de pensamiento que ha sido instaurado por el modelo educativo tradicional a través de los años y que como profesionales continuamos utilizando frecuentemente para evaluar los problemas.

Una de las preocupaciones, en algunas universidades, consiste en que dadas las características de incertidumbre y complejidad del contexto global actual, es evidente la necesidad de superar la división curricular en disciplinas (asignaturas), que fue realizado por cuestiones didácticas y que evidentemente tiene su valor en la organización curricular y en la profundización de los conocimientos disciplinares, sin embargo, esa fragmentación nos aleja de la comprensión del problema en su complejidad y totalidad, por lo que se hace necesario incorporar otros enfoques que entrelacen las disciplinas para construir conocimientos.

Esta precariedad de la educación para dar respuesta a problemáticas tan complejas y cambiantes no es novedad, sin embargo, a pesar de la obviedad, la educación en la mayoría de sus manifestaciones continúa siendo un modelo que replica la estructura gobernante y la fortalece, sin cambios sustanciales en su estilo y modalidad académica, solo con algunos cambios que con frecuencia confunden más de lo que aportan. A nivel formal estos cambios no tienen grandes repercusiones, salvo casos aislados, en ocasiones se asumen por imposición y como una moda más o menos pasajera, en la que pueden incorporarse nombres y conceptos novedosos; sin embargo, en la práctica continúan siendo las mismas modalidades de estructuras pedagógicas y didácticas fragmentadas de hace años.

Tanto en los diseños curriculares, como en las aulas y la mayoría de las capacitaciones docentes formales continúa el asignaturismo, con supremacía de algunas disciplinas sobre otras y falta de integración para observar los fenómenos, procesos y sujetos de manera integral, que posibilite a quien aprende comprender cómo es que se analiza la realidad desde múltiples dimensiones a la vez, y obtener de esta forma una aproximación mayor de la complejidad de lo que se estudia. Además, es común que el proceso de diseño curricular involucre exclusivamente a miembros de la comunidad educativa. Este modo de operar puede influir en que se mantenga una visión parcelada de qué se enseña, cómo se enseña y cuándo. Son estos factores, que se manifiestan en todos los niveles del sistema educativo, los que muestran la dificultad que enfrenta el sistema educativo en su trayecto de transformación hacia un modelo transdisciplinar.

Por otro lado, las teorías pedagógicas han demostrado que los contenidos memorísticos y la desvinculación de los aprendizajes de la realidad no favorecen el aprendizaje. Así como que el centrar la educación en los contenidos en vez de hacerlo en el proceso, es un despropósito. Entre los referentes académicos, Tobón (2005 p.272) plantea que es fundamental que "los estudiantes no repitan de forma literal la definición de los conceptos" y que desarrollen estrategias cognitivas capaces de dar cuenta de la organización y estructura, es decir de procesos.

A pesar de ello, no se han superado por completo tales estructuras en muchos de los sistemas educativos, con excepción de algunos esfuerzos institucionales o individuales. Atendiendo a ello, podrían incluirse cambios en los diseños curriculares que se inclinen hacia la transdisciplinariedad de modo que favorezcan la construcción de conocimientos vinculando a diferentes disciplinas, entrelazándolas en lugar de clasificándolas, que surjan a partir de las problemáticas y que propicien la reflexión con pensamiento crítico para la búsqueda de conocimientos, su selección e interpretación, pensando en el bien común y en el cuidado de todo lo vivo. Ese, por ejemplo, sería un cambio cualitativo en la educación, o en cualquier otra esfera, capaz de remover las anquilosadas estructuras del aparato educativo formal, que tal como está no nos ayuda a ser mejores personas, ni mejores profesionales.

Aún no comenzaba el siglo XXI cuando la UNESCO, atendiendo a los planteamientos de la complejidad expresados por Morin (1999), asume la obra que sería una bisagra para las posturas existentes, "Los siete saberes necesarios para la educación del futuro" y desde su introducción plantea que:

Uno de los desafíos más difíciles será el de modificar nuestro pensamiento de manera que enfrente la complejidad creciente, la rapidez de los cambios y lo imprevisible que caracterizan nuestro mundo. Debemos reconsiderar la organización del conocimiento. Para ello debemos 
derribar las barreras tradicionales entre las disciplinas y concebir la manera de volver a unir lo que hasta ahora ha estado separado (p.7).

Este planteamiento de Morin (1999) deja muy claro que la tarea más difícil, pero a la vez vital, es modificar la forma en la que pensamos y vemos el mundo. El modelo educativo por asignaturas nos ha entrenado en la clasificación, simplificación y descomposición de la realidad para analizarla, estudiarla y solucionarla. El conflicto en esto es que la realidad se presenta compleja y con elementos tratados por las disciplinas ligados entre sí, al descomponerla, se pierde la esencia de lo que se estudia. Además, ese razonamiento reduccionista no está preparado para la incertidumbre y ambigüedad inherente a la complejidad de las situaciones que analiza. El pensamiento que resulta de un modelo educativo transdisciplinario no pretende anular la profundidad de las disciplinas, sino complementarla con la transdisciplinariedad que superaría las barreras entre los saberes uniendo las disciplinas de manera transversal.

Siguiendo esta línea de pensamiento, Espinosa (2007) concibe la transdisciplinariedad como:

Un movimiento de transición paradigmática, que toma el desafío de construir la unidad con la diversidad y que la unidad de la investigación de Edgar Morin se centra justo en la preocupación de un conocimiento no mutilado ni disociado, que pueda respetar lo individual y lo singular insertándolo en su contexto y su conjunto (p.3).

Asumir el enfoque transdisciplinario como el más adecuado para complementar la educación es aceptar la importancia de la totalidad del contexto a la vez que se respeta y considera cada parte. Implica aceptar el cambio de pensamiento y trabajar en estrategias y planes que lo promuevan para dar paso a un tipo distinto de formación profesional. Sobre la imperiosa necesidad de tomar medidas que renueven los procesos de enseñanza aprendizaje y la modalidad evaluativa, son muchos los autores que han evidenciado la preocupación por una realidad que no termina de ser asumida por completo. A modo de ejemplo puede mencionarse a Velásquez (2007, p.30), quien explica que "las condiciones especiales que está tomando el contexto actual en la era de la sociedad del conocimiento y la información conllevan una necesaria transformación de los distintos escenarios en los que se desenvuelven los educandos de todos los niveles y modalidades..."

\section{Solución}

Para lograr cambios significativos en la estructura del modelo educativo y el tipo de pensamiento que se genera a partir de este, se visualiza como una de las formas la generación de espacios de diálogo interactivo y motivador en el ámbito de lo inter y transdisciplinar para reconstruir las formas de pensar. Cabe aquí recuperar la postura de Nicolescu (2002) quien afirma que el instalar el pensamiento complejo y transdisciplinario en las estructuras y programas de la universidad "va a permitir que esta evolucione hacia su misión más importante, algo descuidado actualmente -el estudio de lo universal", postura que coincide con autores como Morin y Espinosa en resaltar la importancia de ver la totalidad y asumir la complejidad de la realidad en todos los ámbitos de estudios.

Si bien en el ámbito educativo, la idea de cambio ha tenido siempre un valor positivo fuertemente ligado a la dimensión utópica o proyectiva de la educación, en la actualidad las innovaciones y los rápidos cambios sociales y tecnológicos constituyen un tema central en la agenda pedagógica. En las universidades, como señala Di Capua (2004, p.17), los estudios en torno a esta temática han sido enfocados "en el currículum formal y en las denominadas 'innovaciones regulatorias' que responden a la lógica arriba-abajo y se circunscriben en general al cambio en los planes de estudio de las distintas carreras universitarias". Sin embargo, los diseños y concepción ideológica de los lineamientos curriculares, así como las prácticas concretas de enseñanza como objeto de innovación no han merecido igual atención por los estudiosos de ese campo, a pesar de que en los discursos circulantes del ámbito académico se apela con frecuencia a conceptos como cambio, reforma, transformación educativa, entre otros. Para instaurar una verdadera transformación educativa a través del modelo transdisciplinario, los cambios deben estar presente en todos los niveles del sistema educativo, para lo cual es de gran importancia, sobre todo, que las estrategias, metodología y planes de enseñanza de los docentes logren inicialmente entrelazar las disciplinas correspondientes a su área de estudio y promuevan el pensamiento complejo en los educandos. De igual manera, el sistema evaluativo tanto del estudiante como del docente debe contemplar estos elementos transdisciplinarios.

Collado (s.f) expresó que "La educación para la ciudadanía mundial promovida por la UNESCO 
en la agenda post-2015 debe contextualizarse multidimensionalmente: desde una perspectiva transnacional, transcultural, transpolítica, transreligiosa y transdisciplinar que tenga en cuenta los diferentes niveles de realidad". La educación superior debe incluir aspectos locales y mundiales, tiene que tocar la cultura, la política, y la religión de una manera integradora. Así como también el diálogo entre el arte y la ciencia, lo que representa el eje de una reunificación entre la cultura científica y la cultura artística. Una universidad trans-formada puede constituir el lugar más adecuado para dar la bienvenida a un nuevo tipo de pensamiento y de acción social que abarque los requerimientos reales que emanan de la sociedad y no los que en las universidades se interpreta que son tales requerimientos. Este es otro elemento importante, los conocimientos transmitidos en las universidades necesitan estar en conexión directa con la sociedad en la que se imparten. Para ello el diseño curricular debe contar con la participación de agentes institucionales del sector educativo, docentes, estudiantes y representantes de la sociedad que bien pudieran ser los futuros empleadores de los profesionales que están en formación. La participación de un grupo diverso permite un diseño curricular más rico, que considera diversas dimensiones de los saberes y que puede promover en su enfoque y su práctica la transdisciplinariedad.

La Red Internacional de Estudios Generales (RIDEG) ha venido desarrollando un proceso de reflexión que ha permitido analizar e incorporar a la discusión cada vez con más fuerza el enfoque transdisciplinario, por considerarlo el más adecuado desde la educación para enfrentar los retos del mundo moderno. Cambiar la concepción tradicional, tanto en la construcción del conocimiento, así como en el hermetismo propio de la academia -que solo da cabida a las voces propias de este espacio- es una labor urgente que está siendo abordada en varias universidades mediante la incorporación de un enfoque interdisciplinario en algunas y transdisciplinario en otras y el ejercicio de investigaciones en esta misma línea que a través de la integración de conocimientos convocan a actores de variedad de disciplinas y participantes que no pertenecen al mundo de la academia para intentar abordar la mayor cantidad de dimensiones de la vida.

Para quienes consideran la transdisciplinariedad como la mejor opción para dar respuesta a las necesidades del mundo actual, tienen la convicción de que desde esta se favorece el abordaje, en la docencia, de aquellas problemáticas o contenidos de actualidad de una manera mucho más abarcadora que desde otros enfoques, debido a que, según Nicolescu (1996: 37)

La Transdisciplinariedad comprende, como el prefijo "trans" lo indica, lo que está, a la vez, entre las disciplinas, a través de las diferentes disciplinas y más allá de toda disciplina. Su finalidad es la comprensión del mundo presente, y uno de sus imperativos es la unidad del conocimiento.

La transdisciplinariedad es una realidad a la que se enfrentan los profesionales en el ejercicio de sus carreras. El choque está cuando el tipo de pensamiento que se ha desarrollado durante la etapa educativa no es capaz de dar respuesta a la realidad que se presenta en el mundo laboral. Este es un factor muy importante para tomar en cuenta, la transdisciplinariedad enriquece la sociedad, favorece su desarrollo y facilita la generación de soluciones a sus problemas. Al respecto puede considerarse que:

La transdisciplinariedad es el nivel más profundo de colaboración alcanzable por equipos de diferentes expertos, a los que se pueden agregar actores de interés con conocimiento local sobre el sistema. En el marco transdisciplinar, investigadores de experiencias diversas trabajan conjuntamente para responder a una problemática que ellos mismos han definido bajo un marco conceptual; este enfoque rompe con las barreras disciplinares debido a que el lenguaje compartido y las metodologías de solución de problemas son desarrolladas [en conjunto] (Ciannelli, 2014, p. 1048).

En todos los niveles de la educación es posible obtener una muestra de las diversas disciplinas de manera independiente una de otra. Como consecuencia, el resultado es una "mente fragmentada" o "semi- especializada" y refleja un conocimiento no tan equilibrado como ocurría en la etapa de artes liberales, aunque los profesionales en cada área pueden leer, conocer algo de historia y apreciar pinturas y música. Velencei, Baracskai, Dorfler y Stierand (2016) afirman que la educación formal que produce espíritus cultivados semi-especializados es a la vez necesaria e inútil. La necesidad surge porque esa es la forma como los futuros profesionales aprenden a pensar según la lógica de sus disciplinas específicas. 
Al mismo tiempo, esta educación disciplinaria enfocada puramente en lo intelectual es también inútil, ya que no permite a estos futuros profesionales "comprender la lógica contextualmente sensible de la praxis que es capaz de manipular magistralmente conceptos de libros de texto" (Velencei, Baracskai, Dorfler y Stierand, 2016).

Barrantes (2015) plantea que:

Se evidenció el hecho de que el paradigma positivista que ha influido en tantas áreas del saber, pero - de manera particular- en la visión de las disciplinas científicas como campos inconexos y que ha llevado a muchos profesionales académicos a radicalizar posiciones de enclaustramiento en sus especializaciones, debe ser sustituido por otro de índole sistémica, que le permita a las nuevas generaciones de profesionales en docencia establecer relaciones entre datos, informaciones y opiniones de diversa naturaleza y que, en un esfuerzo inter y transdisciplinario, realicen un proceso de síntesis vital, lo cual sea una práctica en la organización de sus ideas para que desarrollen y enseñen a desarrollar proyectos de vida coherentes y con propósito (p.8).

La tendencia entonces sugiere un desprendimiento del pensamiento simple y la profundización en el pensamiento complejo, de manera que la nueva concepción influya en la forma de hacer ciencia e investigación. La perspectiva de la complejidad implica indagar en la relación entre sujeto/objeto, todo/parte y la noción de tiempo e historia, en el análisis, búsqueda y construcción de soluciones. Visto así, puede pensarse que la separación de la ciencia en básica y aplicada constituye una barrera que dificulta la colaboración entre las mismas. Los "problemas sociales" y su grado de urgencia y necesidad provocan que los programas de investigación adopten necesariamente una forma de trabajo fuera del campo disciplinar; es decir, se crean prácticas científicas o de investigación que trascienden el encuadre disciplinar (Beltrán, 2016). En las ciencias, tanto el enfoque multidisciplinario como interdisciplinario han ido realizando aproximaciones a una nueva forma de abordaje y es probable que la transdisciplinariedad responda a la necesidad de perspectivas científicas más amplias al momento de enfrentar problemáticas complejas (Ciannelli, et. al., 2014), para lo cual se requiere contar con más documentación y experiencia en las universidades a fin de que se contrasten estas opiniones.

La educación transdisciplinaria se presenta como una alternativa a la perspectiva de que la ciencia y la tecnología son la solución para todos los problemas de la humanidad. Independientemente de los avances hechos en estas, la educación debería implementar estrategias de balance. La ciencia y la tecnología por sí solas no contemplan las decisiones morales, espirituales o éticas, por mencionar algunas, "éstas son simplemente herramientas para la invención y creación de herramientas" (Yeh, 2015, p.4). Es el profesional y su conjunto de conocimientos, habilidades y destrezas lo que definen la calidad de la solución a los problemas, y es la educación superior la que a través de su modelo educativo define el tipo de profesional y su capacidad para responder adecuadamente a los retos de un mundo globalizado. De allí que se considera imperante el establecimiento del enfoque transdisciplinario en la educación superior para generar un nuevo perfil del profesional de la ciencia y la tecnología, un perfil que incluya las herramientas necesarias para resolver problemáticas complejas. (Lemarchand, citado en Beltrán, 2016).

\section{Propuesta transdisciplinar para docencia, investigación y evaluación}

La incorporación de docencia e investigación transdisciplinar son una necesidad que ha llevado a algunas universidades a abrir la discusión sobre el tema, a hacer investigación acción con sus docentes, a implementar estrategias y herramientas de evaluación transdisciplinarias e incluso a abrir espacios a los docentes de diferentes disciplinas para compartir sus prácticas exitosas y generar proyectos integradores. Estos acercamientos posibilitan comparar y compartir las prácticas transdisciplinarias entre distintas universidades, así como identificar los mayores logros en cada una de ellas aplicando este tipo de docencia. Estos avances son pequeños pasos que todavía requieren de mayor análisis y rigor metodológico, aun así, son esfuerzos importantes que ayudan a pensar la educación superior desde una perspectiva integradora.

El estudio de Osorio (2016) intenta mostrar una propuesta que incluye elementos de transdisciplinariedad en el currículo de una institución educativa, con el propósito de establecer conexiones que promuevan en los estudiantes el acercamiento, 
deconstrucción, reconstrucción y construcción de un conocimiento más significativo. Este foco investigativo arrojó resultados que muestran discrepancia entre lo que se dice y se cree saber y lo que se hace en el aula por parte de los docentes. Este resultado nos permite dirigir la mirada hacia la importancia de que los cambios no solo sean a nivel estructural, sino que los docentes estén conscientes y capacitados en lo que se supone que el currículo de la institución promueve, como se evidenció en esta investigación, ese no siempre es el caso, a veces el currículo está redactado con un enfoque transdisciplinario mientras la realidad en las aulas es otra. La propuesta de Osorio (2016) busca aportar una mirada distinta al ejercicio educativo a través de "la reestructuración del plan de estudios, romper el tradicional 'asignaturismo' y posibilitar que los profesores establezcan puentes temáticos/problemáticos entre las diferentes áreas del saber, desde una óptica global sin dejar de lado lo local" (p.54).

Cabe señalar que el proceso académico que incorpora la transdisciplinariedad en la docencia y la investigación no surge espontáneamente y que para alcanzar la aplicación concreta de la transdisciplinariedad en la docencia se requiere contar con la voluntad y convicción de las instancias institucionales, la sensibilización y capacitación del cuerpo docente y estrategias didácticas apropiadas.

Además de la capacitación docente en la construcción de planes y estrategias educativas transdisciplinarias son necesarias la elaboración e implementación de herramientas que permitan evaluar y documentar las prácticas docentes de acuerdo a los criterios que deben estar presentes en la docencia transdisciplinaria; es a través de estas herramientas que se pueden hacer los ajustes necesarios para cumplir el objetivo de cambio.

En relación con la evaluación docente, resulta evidente considerar que esta tiene que coincidir con la práctica transdisiciplinaria que se pretende establecer, y partir de cómo se espera que se manifieste en el proceso de enseñanza aprendizaje, así como de las capacidades y atributos primordiales que el docente debe implementar y desarrollar. En el caso de la docencia transdisciplinaria, por no estar establecida formalmente de manera generalizada, se carece de información suficiente que esté sistematizada y con rigor científico, sin embargo, se cuenta con algunos diseños de herramientas de evaluación propias para este tipo de docencia. Mientras más universidades asuman el desafío de implementarla aun en forma piloto, se logrará avanzar y será posible definir y contrastar perfiles transdisciplinarios y metodologías validadas para evaluar la calidad de las prácticas docentes y su adherencia a la transdisciplinariedad.

Lo mismo ocurre con la investigación, la limitación de las disciplinas para afrontar los problemas sociales cada vez más complejos e impredecibles van orientando la investigación a trascender los límites disciplinares, con diseños que no pueden inscribirse en los encuadres disciplinares (Gibbons, et al. 1997). Las problemáticas actuales que podemos englobar en las relacionadas con género, ecología, interacción entre los sistemas bio-ecológicos y sociales, el desarrollo sustentable, y que siguen emergiendo, algunas de reconocida relevancia para la UNESCO, y que las instituciones de educación superior deben atender, demandan un enfoque integrador y que en esta propuesta se sugiere el transdisciplinar como idóneo para docencia, investigación y evaluación. "Las comisiones de evaluación de los científicos siguen manteniendo las mismas estructuras disciplinares de hace 50 años. Prácticamente no existen comisiones evaluadoras con perfiles interdisciplinarios o transdisciplinarios y tampoco existen aún, metodologías estandarizadas para poder analizar la calidad y pertinencia de este tipo de investigaciones" (Lemarchand, citado en Beltrán, 2016. p.126). Este estado inmóvil en el ámbito de la investigación y la ciencia representa una barrera en el cambio que necesita la educación superior, no importa si cambian algunas estructuras, si aun en aspectos como la investigación y la ciencia se siguen considerando válidos solo los procedimientos heredados de la concepción fragmentada del conocimiento, incluso en el aspecto evaluativo.

Por su parte, hay pensadores como Barrantes (2015) que defienden la postura de que "las nuevas generaciones de docentes deben formarse mediante el paradigma de la complejidad, (Morín 2007) es decir, con un enfoque inter y transdisciplinario, el cual asuman como propio en sus vidas y lo transmitan a sus educandos" (p.2).

Queda claro que capacitación y evaluación de los profesionales académicos juega un rol muy importante en la implementación de cualquier paradigma educativo en general y en este caso, de la educación transdisciplinaria en particular. No se trata solo 
de reconocer la vitalidad de la transdisciplinariedad en la educación, sino de asumirla y practicarla junto con el grupo de estudiantes. Implica un cambio de mentalidad y de forma de construir conocimientos, así como el involucrar a todos los niveles del sistema educativo y a representantes de la sociedad en este nuevo enfoque. En él, el desarrollo profesional de cada docente y la evaluación de su desempeño deben estar ligados al camino de la transdisciplinariedad, de otra forma, aunque la institución teóricamente proclame en su currículum la transdisciplina en la docencia y la investigación, sin estos componentes se dificulta afirmar su implementación. Como plantea Dieleman (2014):

En el campo de la didáctica de enseñar y las capacidades correspondientes de los profesores, la hermenéutica transdisciplinaria tiene muchas implicaciones. La sensibilidad, las capacidades de autoconocimiento, reflexión e introspección que son requeridas de los estudiantes, obviamente tienen que ser mostradas por parte de los profesores (p.467).

A fin de lograr que cada docente pueda incorporar y modelar elementos transdisciplinarios en su práctica, este tiene que conocerlos y adoptarlos primero. La capacitación no es opcional, sino necesaria y la evaluación del desempeño también es vital para garantizar una práctica educativa transdisciplinaria de calidad. Se requiere de un docente genuinamente transdisciplinario para formar a un profesional transdisciplinario.

\section{Conclusión}

Para concluir, asumir un enfoque diferente para la gestión docente demanda sensibilizar al profesorado respecto a las razones por las cuales las problemáticas del mundo actual desbordan cualquier propuesta académica convencional. Así también es necesario motivar sobre las bondades de un enfoque como el transdisciplinar que supera las fronteras disciplinares con sustento epistemológico y que en la práctica logre acercamientos en todas las variantes que sea necesario y genere procesos colectivos de aprendizaje continuo. Docentes empoderados de una modalidad transdisciplinar de su práctica van a requerir diseños evaluativos para su desempeño, plasmados en rúbricas e instrumentos que deberían aplicarse por un equipo transdisciplinar. Estas evaluaciones deberían recoger los criterios para categorías y subcategorías identificadas para este tipo de docencia, de manera que cada docente conozca con precisión qué se espera de su práctica, que estaría siendo evaluado de manera permanente y que él mismo sería uno de los miembros del equipo evaluador.

Otra perspectiva por considerar es que, aunque la meta de la enseñanza transdisciplinaria es formar investigadores/profesionales con una visión integradora más allá de las disciplinas, esto no significa que se elimina la formación monodisciplinar, o que se desvaloriza la formación disciplinar, por el contrario, se reconoce su gran importancia e incluso que es indispensable contar con especialistas disciplinares para aportar a la transdisciplinariedad.

Por otro lado, una manera de contribuir a contrarrestar los efectos negativos de la globalización económica y la complejidad e incertidumbre del mundo actual es promoviendo una universidad transformada con un cambio de mirada a través de la educación transdisciplinar. El cambio de mirada envuelve "la aceptación de que el conocimiento puede y debe construirse no solo en los ámbitos disciplinarios, sino también en los márgenes del sistema, en los territorios de frontera, allí donde florecen los encuentros más fecundos en lo que podríamos denominar 'los ecotonos del saber'” (Novo, 2009, citada en Beltrán, 2016 p.147). Aun cuando a nivel curricular se han hecho algunos esfuerzos por transitar hacia un saber integrado que supere las barreras disciplinares, da la impresión de que, a nivel de capacitación, prácticas docentes y evaluación no se han logrado tantos cambios, o al menos no resultan tan evidentes.

Finalmente, en estas reflexiones se reconoce de suma importancia acompañar este camino hacia la inter y transdisciplinariedad en la educación superior con el desarrollo e implementación de capacitación docente y herramientas de evaluación que monitoreen y garanticen la calidad del proceso de enseñanza-aprendizaje considerando los elementos y criterios que la caracterizan. En definitiva, es necesario el desarrollo de una educación general inter y transdisciplinaria para lograr la transformación y fortalecimiento de la universidad en el siglo XXI que debe superar la segmentación del saber y tener como propósito el estudio de lo universal y del ser humano integral, no fragmentado y en su contexto. Por ello se reconoce como una posibilidad para fortalecer a la universidad en nuestros días la necesidad de una educación general transdisciplinaria (Vélez 2014). 


\section{Referencias bibliográficas}

Barrantes-Montero, L. G. (2015). Cómo orientar la formación del personal docente nuevo hacia la adquisición de un paradigma sistémico. Revista Electrónica Educare, 19(3), 1-10.

Beltrán, D. (2016). La evaluación de la actividad universitaria y la validación del conocimiento académico: presupuestos epistemológicos desde el MODO 2 de producción del conocimiento. Revista de Epistemología y Ciencias Humanas, 8, 131-152.

Ciannelli, L., Hunsicker, M., Beaudreu, A., Bailey, K., Crowder, L; Finley, C. y Chingbu, P. (2014). Transdisciplinary graduate education in marine resource science and management. ICES Journal of Marine Science. 71(5), p. 1047-1051. Disponible en: https://academic.oup.com/icesjms/ article/71/5/1047/648379

Collado J. (s.f). Los Fundamentos Astrobiológicos de la Humanidad: el Origen de la Consciencia Cosmoderna.GlobalEducationMagazine. Disponible en:http://www.globaleducationmagazine.com/ los-fundamentos-astrobiologicos-de-la-humanidadel-origen-de-la-consciencia-cosmoderna/

Di Capua, A. (2004). Las reformas curriculares en los planes de formación de profesores en la Universidad. Facultad de Filosofía y letras. Universidad de Buenos Aires

Dieleman, H. (2014). Hacia una hermenéutica transdisciplinaria para arquitectos y diseñadores de paisaje. IV Congreso Internacional de Ciencia y Arte del Paisaje. En: Paisajes Alternativos (2016) Acampa. Jalisco, México.

Espinosa, A. (2007). Presentación. Visión Docente Con-ciencia. Año VI Nro. 34. Arkos. Puerto Vallarta. Jalisco.

Gibbons, M., Limoges, C., Nowotny, H., Schwartzman, S., Scott, P. y Trow, M. (1997). La nueva producción del conocimiento. Barcelona: Ediciones PomaresCorredor.

Morin, E. (1999). Los siete saberes necesarios para la educación del futuro. UNESCO

Morin, E. (2007). Introducción al pensamiento complejo. Valladolid: Gedisa Editorial. En:http:// www. pensamientocomplejo.com.ar/docs/files/ MorinEdgar_Introduccionalpensamiento-complejo_ Parte1.pdf

Nicolescu, B. (1996). La Transdisciplinariedad. Manifiesto. México: Multiversidad Mundo Real Edgar Morín, A.C.
Osorio, J. (2016). Educandos y su tránsito por el mundo complejo: elementos de la transdisciplinariedad en el currículo de una institución educativa del municipio de Palmira. Revista de Investigaciones UCM, 16 (27), 54-66.

Pozzoli, M.T. (2006). El sujeto de la complejidad: La construcción de un Modelo Teórico Transdisciplinar (eco-psico-socio-histórico-educativo). Polis Revista Latinoamericana. Disponible en: https:// journals. openedition. org/polis/4921

Red Internacional de Estudios Generales (RIDEG) En: https://www.rideg.org/

Rondelli, B. (2015). Transdisciplinaryresearch projects: a matter of evaluation oreducation? SIRIS academics. Disponible en: http://www. sirisacademic.com/wb/ blog/transdisciplinary-research-projects-a-matter-ofevaluation-or-formation/

Tobón, S. (2005). Formación basada en competencias. Pensamiento complejo, diseño curricular y didáctica. España: Universidad Complutense de Madrid.

Velásquez, J. (2007). Los nuevos ambientes del aprendizaje, un imperativo para la educación del siglo XXI. Visión Docente Con-ciencia. Año VI Nro. 34. Arkos. Puerto Vallarta. Jalisco

Velencei, J., Baracskai, Z., Dorfler, V. y Stierand, M. (2016). Supporting the Competent Practitioner: Trans-disciplinary Coaching with a KnowledgeBased Expert System. International Journal of Management Science and Business Administration. Vol. 2, N. ${ }^{\circ}$ 12, Pags. 20-27. Disponible en: http://dx.doi.org/10.18775/ ijmsba.1849-5664-5419.2014.212.1002

Vélez, W. (2014). Los nuevos retos de la Educación General universitaria en el siglo XXI. Revista Nuevo Humanismo. Vol.2 Núm.2, Disponible en:http://www.revistas.una.ac.cr/index.php/ nuevohumanismo/article/view/7207

Yeh, R. (2015). Convergence of Transdisciplinary Education [Convergencia de la Educación Transdisciplinaria]. Journal of Integrated Design and Process Science. 19(4), p. 3-8. Disponible en:http://eds.b.ebscohost.com/ehost/pdfviewer/ pdfviewer? sid=25f4bb43-1e0c-4d82-a222-dc3c9 10e0615\%40sessionmgr103\&vid=0\&hid=114 